matical literature sadly reminds us; but one who like Sturm can seize on the important and simple modifications of a given problem has certainly one of the most essential elements of mathematical greatness.

Harvard UNIVERSity,

Cambridge, Mass.

A SENSUOUS REPRESENTATION OF PATHS THAT

LEAD FROM THE INSIDE TO THE OUTSIDE OF AN ORDINARY SPHERE IN POINT SPACE OF FOUR DIMENSIONS WITHOUT PENETRATING THE SURFACE OF THE SPHERE.

BY PROFESSOR C. J. KEYSER.

(Read before the American Mathematical Society, April 28, 1911.)

THE logical or analytic existence of such paths-their existence in and for thought as distinguished from intuition or imagination-has been long familiar to every one, and may be made evident even to a freshman, so simple is the sufficient algebraic argument. But all efforts to envisage the paths are defeated completely.

It is the purpose of this note to show how the existence of the paths may be made evident to the intuition and even to the senses of sight and touch. The purpose is achieved by a simple transformation correlating the points of 4-space $S_{4}$ with the spheres of ordinary space $S_{3}$, including all spheres of real center and pure imaginary radius. In this way unintuitable situations in $S_{4}$, like that presented by the paths in question, are represented by intuitable analytic equivalents in $S_{3}$, and these equivalents may be rendered sensible by easily constructible physical models.

The simplest possible correlation of the kind in question is that in which the point $(x, y, z, w)$ of $S_{4}$ and the sphere (of $S_{3}$ ) having $(x, y, z)$ for center and $\sqrt{ } w$ for radius.shall be a pair of correspondents.

The representative in $S_{3}$ of a lineoid (an ordinary 3-space) $A x+B y+C z+D w+E=0$ of $S_{4}$ is a linear complex of spheres such that, if $\left(x_{1}, y_{1}, z_{1}, w_{1}\right)$ be a point of the lineoid, the 
corresponding sphere of the complex is the sphere that has its center at $\left(x_{1}, y_{1}, z_{1}\right)$ and its radius equal to $\sqrt{ } w_{1}$. Those spheres of the complex that have a same radius, $r=\sqrt{ } w$, have for locus of their centers the plane $A x+B y+C z+D r^{2}+E=0$; and the planes that thus correspond to different values of $r$ are parallel.

In particular, the lineoid $w=k>0$ which is parallel to the $x y z$-lineoid of reference, and on the positive side of it, is represented in $S_{3}$ by the sphere complex consisting of the real spheres whose center-locus is the totality of points of $S_{3}$ and all of which have the same radius $r=\sqrt{ } k$ : a complex naturally to be regarded as parallel to the sphere complex composed of the points of $S_{3}$ conceived as spheres of zero radius.

The line of $S_{4}$ determined by the points $\left(x_{1}, y_{1}, z_{1}, w_{1}\right)$, $\left(x_{2}, y_{2}, z_{2}, w_{2}\right)$ is represented in $S_{3}$ by a range of spheres determined by the two spheres that correspond respectively to the given points. The locus of the centers of these spheres is the line determined by the points $\left(x_{1}, y_{1}, z_{1}\right),\left(x_{2}, y_{2}, z_{2}\right)$. A sphere of the range whose center is $(x, y, z)$, where

$$
\begin{gathered}
x=\left(x_{1}+\lambda x_{2}\right) /(1+\lambda), y=\left(y_{1}+\lambda y_{2}\right) /(1+\lambda), \\
z=\left(z_{1}+\lambda z_{2}\right) /(1+\lambda),
\end{gathered}
$$

has for radius $r=\sqrt{ } w$, where $w=\left(w_{1}+\lambda w_{2}\right) /(1+\lambda)$; so that in general the size of a sphere of a range varies as its center moves along the locus of centers of the range, i. e., as the sphere moves along the range, generating the range as the path of a moving sphere just as in $S_{4}$ the line corresponding to the range in $S_{3}$ is the path of a moving point.

The 4-dimensional sphere $x^{2}+y^{2}+z^{2}+w^{2}-R^{2}=0$ of $S_{4}$ is represented in $S_{3}$ by a certain quadratic complex of spheres, namely, that whose spheres have for locus of centers the ensemble of points within or on the sphere $x^{2}+y^{2}+z^{2}-R^{2}=0$ and whose radii as the distance of the center from the origin increases vary for $w$ positive from $\sqrt{ } R$ to zero and for $w$ negative from $i \sqrt{ } R$ to zero. The spheres of the complex fall into two equal classes according as the radii are real or pure imaginary.

The pair of equations

$$
\begin{aligned}
& x^{2}+y^{2}+z^{2}+w^{2}-R^{2}=0, \\
& w=k \quad(0<k<R),
\end{aligned}
$$


determine an ordinary sphere (of $S_{4}$ ) that is immersed in the lineoid $w=k$. This sphere is represented in $S_{3}$ by a quadratic congruence of spheres, which may be called a sphered sphere. It is composed of real (visualizable) spheres whose centers have for their locus the sphere $x^{2}+y^{2}+z^{2}=R^{2}-k^{2}$ and whose radii are $\sqrt{ } k$. This sphered sphere is immersed in the sphere complex corresponding, as above seen, to the lineoid $w=k$.

A point $\left(x_{1}, y_{1}, z_{1}, w_{1}\right)$ of $S_{4}$ is inside, on, or outside of the sphere (1) according as $x_{1}^{2}+y_{1}{ }^{2}+z_{1}{ }^{2}<,=$, or $>R^{2}-w_{1}^{2}$, provided $w_{1}=k$. A point whose $w \neq k$ is properly neither inside, nor on nor outside the sphere (just as in ordinary space a point external to a given plane is properly neither inside, nor on, nor outside of a given circle of the plane). According as a point of $S_{4}$ is inside, on, or outside of the sphere (1), or has none of the three relations to it, so in $S_{3}$ the sphere that represents the given point is respectively inside, on, or outside the sphered sphere representing the sphere (1), or has none of the three relations to the sphered sphere.

Let $\left(x_{1}, y_{1}, z_{1}, k\right)$ and $\left(x_{2}, y_{2}, z_{2}, k\right)$ be two points respectively inside and outside of the sphere (1). The condition that a point

$$
\begin{gathered}
x=\left(x_{1}+\cdot \lambda x_{2}\right) /(1+\lambda), \quad y=\left(y_{1}+\lambda y_{2}\right) /(1+\lambda), \\
z=\left(z_{1}+\lambda z_{2}\right) /(1+\lambda), \quad w=k,
\end{gathered}
$$

of the line determined by the given points shall be on the sphere (1) has a positive discriminant, showing that the line pierces the sphere in two real points. This case is the familiar one that occurs in the point geometry of $S_{3}$ and thus lies in the field of geometric intuition. In the sphere geometry of $S_{3}$ the case is represented by a sphere range determined by two spheres having $\sqrt{ } k$ for radii and situated respectively inside and outside of the sphered sphere corresponding to sphere (1). It is essential to note that all the spheres of the range are of the same size and that the size is that of the spheres composing the sphered sphere, so that, as a sphere traverses the range, it twice coincides with a sphere of the sphered sphere; that is, the range as a path of a moving sphere twice penetrates the (surface of the) sphered sphere.

Now consider the lines of $S_{4}$ determined respectively by the point pairs $\left(x_{1}, y_{1}, z_{1}, k\right),\left(x_{3}, y_{3}, z_{3}, w_{3}\right)$, and $\left(x_{3}, y_{3}, z_{3}, w_{3}\right)$, $\left(x_{2}, y_{2}, z_{2}, k\right)$, where $w_{3}>$ or $<k$, say $w_{3}>k$. No point of the 
former line is on sphere (1), for such a coincidence of points requires that $\left(k+\lambda w_{3}\right) /(1+\lambda)=k$, a condition that is satisfied only when $\lambda=0$, which yields the point $\left(x_{1}, y_{1}, z_{1}, k\right)$, but this point is by hypothesis not on but inside sphere (1). For a like reason no point of the latter line is on sphere (1). Hence the broken line running from the first to the second and from this to the third of the given points is a path (for points) leading from the inside to the outside of sphere (1) without penetrating the surface. It is such analytically evident but unintuitable paths whose existence is to be made evident to spatial intuition and to sight and touch. The means thereto is now obvious. In $S_{3}$ the two spheres representing the first and last of the given points are respectively inside and outside of the sphered sphere representing sphere (1). The sphere representing the point $\left(x_{i}, y_{3}, z_{3}, w_{3}\right)$ is neither inside nor on nor outside of the sphered sphere, a fact intuitively manifest because the sphere in question is larger than the spheres $(r=\sqrt{ } k)$ composing the sphere complex in which the sphered sphere is immersed. As to the broken sphere range representing the broken line, it is intuitively evident that it does not penetrate the sphered sphere (whose spheres are all of radius $\sqrt{ } k$ ) for the spheres composing the first part of the broken range increase in size from that of the initial sphere (of radius $\sqrt{ } k$ and lying inside of the sphered sphere) up to the final sphere (radius $\sqrt{ } w_{3}$ ) of that part, whilst the spheres of the second part decrease in size from that of the sphere just mentioned down to the final sphere of the range, this sphere being outside of the sphered sphere and having $\sqrt{ } k$ for radius.

To construct a model rendering the matter sensibly evident it is plainly sufficient (1) to distribute throughout a small region of space a small number of small spheres, fixing them in position as by slender vertical steel rods, some of the spheres being of equal radius $r$, one or more of greater radius and one or more of less, some of the spheres intersecting (though this is not indispensable) and two or more of them, if transparent, being concentric (though this feature may be omitted); (2) to construct a spherical frame (say of wire) that shall include one or more of the spheres of radius $r$ and exclude some of them, and to locate a number of spheres of radius $r$ so that their centers shall be points of the frame and be suitably distributed upon it; (3) to join by a rod the centers of two spheres of radius $r$, one of them inside the frame, the other outside, and to place 
a number of spheres of radius $r$ so that their centers shall be on the rod and be suitably distributed on it; (4) finally to join by a rod the center of a sphere of radius $r$ inside the frame with the center of a sphere of radius $>r$ (it might as well be $<r$ ) and by a rod the center of the latter sphere with a sphere of radius $r$ outside the frame, and, then, to locate a number of spheres so that the centers of some of them shall be on one of the rods and those of the rest on the other, the spheres on the former rod suitably increasing in size as their centers approach that of the sphere common to the two rods, whilst the spheres on the second rod suitably decrease in size as their centers recede from the common point of the rods.

The model obviously enables one to distinguish by sight or by touch a sphere range representing a line of $S_{4}$ that joins a point inside of an ordinary sphere to an outside point without penetrating the surface from a sphere range representing the case where penetration occurs.

Columbia University, April, 1911.

\section{A DIRECT PROOF OF THE THEOREM THAT THE NUMBER OF TERMS IN THE EXPANSION OF AN INFINITE DETERMINANT IS OF THE SAME POTENCY AS THE CONTINUUM.}

BY DR. N. J. LENNES.

(Read before the American Mathematical Society, February 25, 1911.)

THE following rather obvious theorem is thought of sufficient interest to warrant the publication of a direct proof.

THEOREM: The number of terms in an infinite determinant is of the potency $c$ of the continuum.

Proof. It is well known that every infinite determinant can be brought into the form

$$
\left|\begin{array}{cccccc}
a_{11} & a_{12} & a_{13} & \cdots & a_{1 n} & \cdots \\
a_{21} & a_{22} & a_{23} & \cdots & a_{2 n} & \cdots \\
\cdot & \cdot & \cdot & \cdots & \cdot & \cdots \\
a_{n 1} & a_{n 2} & a_{n 3} & \cdots & a_{n n} & \cdots \\
\cdot & \cdot & . & \cdots & \cdot & \cdots
\end{array}\right|
$$

